Supporting Information for

\title{
Preferential Interactions of a Crowder Protein with the Specific Binding Site of a Native Protein Complex
}

Xu Dong ${ }^{\dagger, \#}$, Ling-Yun Qin ${ }^{\dagger, l, \#, ~ Z h o u ~ G o n g ~}{ }^{\dagger}$, Sanbo Qin ${ }^{\S, \perp}$, Huan-Xiang Zhou ${ }^{*, \S, \perp}$, Chun Tang ${ }^{*, \star}$

${ }^{\dagger}$ Center for Computational Biology, Academy for Advanced Interdisciplinary Studies, Innovation Academy for Precision Measurement Science and Technology, Chinese Academy of Sciences, Wuhan, Hubei 430071, China;

*Beijing National Laboratory for Molecular Sciences, College of Chemistry and Molecular Engineering, and Peking-Tsinghua Center for Life Sciences, Center for Computational Biology, Academy for Advanced Interdisciplinary Studies, Peking University, Beijing 100871, China;

$\S$ Department of Chemistry and Department of Physics, University of Illinois at Chicago, Chicago, Illinois 60607, United States;

'University of Chinese Academy of Sciences, Beijing 100049, China;

${ }^{\perp}$ Institute of Molecular Biophysics, Florida State University, Tallahassee, FL 32306, United States

*Email: tang_chun@pku.edu.cn

*Email: hzhou43@uic.edu

\#Equal contributions 


\section{Experimental Section}

\section{Protein sample preparation}

The genes encoding HPr and EIN (the N-terminal domain of enzyme I including residues 1-249) were cloned to the pET11a vector. Cysteine mutations, including E5C, E25C and E66C of HPr, were introduced using the QuikChange approach. BL21* cells were used for expressing the proteins. Uniformly ${ }^{15} \mathrm{~N}$-labeled proteins were prepared in $\mathrm{M} 9$ minimum medium, with ${ }^{15} \mathrm{~N}$ $\mathrm{NH}_{4} \mathrm{Cl}$ as the sole nitrogen source. Uniformly $\left[{ }^{2} \mathrm{H},{ }^{15} \mathrm{~N}\right]$-labeled proteins were expressed in M9 medium with D-glucose-1,2,3,4,5,6,6- $\mathrm{d}_{7}$ as the sole carbon source; cell culture was diluted three times before IPTG induction. The proteins were purified using FFQ anion exchange columns and Superdex-75 gel-filtration columns (Cytiva) in tandem. Purified proteins were verified using SDS gel and electrospray mass spectrometry (Bruker Daltonics).

Purified proteins were buffer-exchanged (Amicon Ultra) into $20 \mathrm{mM}$ Tris $\bullet \mathrm{HCl}$ buffer at pH 7.4 containing $150 \mathrm{mM} \mathrm{NaCl}$. For macromolecular crowding, the diluted protein solution was carefully mixed with Ficoll-70 (Sigma-Aldrich, Cat\# 72146-89-5) or BSA (Sigma-Aldrich, Cat\# 9048-46-8) at desired weight/volume (w/v) ratios. The cysteine mutants of HPr were prepared in the same buffer with the addition of $2 \mathrm{mM}$ DTT.

\section{NMR titrations and relaxation measurements}

Unless otherwise indicated, NMR spectra were acquired at $313 \mathrm{~K}$ on a Bruker $600 \mathrm{MHz}$ spectrometer equipped with a cryogenic probe. The elevated temperature allowed for wellresolved and sharp NMR peaks, even in the presence of the crowders. The NMR buffer was 20 $\mathrm{mM}$ Tris $\bullet \mathrm{HCl}$ at $\mathrm{pH} 7.4$ containing $150 \mathrm{mM} \mathrm{NaCl}$, with the addition of $10 \% \mathrm{D}_{2} \mathrm{O}$. A series of ${ }^{1} \mathrm{H}^{15}{ }^{15}$ HSQC spectra were recorded to monitor the chemical shift perturbations (CSPs) of 100 $\mu \mathrm{M}{ }^{15} \mathrm{~N}$-labeled $\mathrm{HPr}$ upon titrating with unlabeled EIN, at concentrations of $10,25,50,75,100$, 125,150 , and $200 \mu \mathrm{M}$. The protein samples were prepared either in the buffer, or in the 
presence of $10 \% \mathrm{w} / \mathrm{v}$ macromolecular crowder. The HSQC spectra were processed using NMRPipe, ${ }^{1}$ and CSPs (Figures 1 and 6) were calculated as $\left(0.5 \times(\delta \mathrm{H})^{2}+0.1 \times(\delta \mathrm{N})^{2}\right)^{0.5}$, where $\delta \mathrm{H}$ and $\delta \mathrm{N}$ were the chemical shift changes in the ${ }^{1} \mathrm{H}_{\mathrm{N}}$ and ${ }^{15} \mathrm{~N}$ dimensions. For the assessment of soft interactions between BSA and $\operatorname{HPr}, 200 \mu \mathrm{M}\left[{ }^{2} \mathrm{H},{ }^{15} \mathrm{~N}\right]$-labeled $\mathrm{HPr}$ was titrated with $10 \%$, $15 \%, 20 \%$, and $30 \%$ w/v BSA. The $K_{\mathrm{D}}$ values for HPr-EIN (Figure 1) and HPr-BSA (Figure 6D) interactions were obtained by fitting the CSPs with a single-site binding model over the concentration of the titrant protein, with the uncertainties of the fits also reported.

For measuring the relaxation rates of amide nitrogen atoms, the samples included: (1) 300 $\mu \mathrm{M}{ }^{15} \mathrm{~N}$-labeled HPr; (2) $300 \mu \mathrm{M}\left[{ }^{2} \mathrm{H},{ }^{15} \mathrm{~N}\right]$-labeled EIN; and (3) $300 \mu \mathrm{M}{ }^{15} \mathrm{~N}$-labeled HPr mixed with equimolar unlabeled EIN. For crowded conditions, $10 \%$ w/v Ficoll-70 or 10\% w/v BSA was added. The longitudinal relaxation experiments were performed at recovery delays of 0.01 , $0.1,0.2,0.35$, and $0.5 \mathrm{~s}$. The transverse relaxation rates $\left(R_{2}\right)$ were measured with refocusing delays of $16.97,50.91,101.82,152.73$, and $203.64 \mathrm{~ms}$. The $R_{1}$ and $R_{2}$ relaxation rates were obtained from fitting the relative peak intensities with single-exponential decays; the errors for $R_{1}$ and $R_{2}$ were the uncertainties of the fits, and the errors for the ratio and product of $R_{2}$ and $R_{1}$ were propagated errors.

\section{Measurement of intermolecular PREs}

After removing DTT from the protein buffer by desalting, isotopically unlabeled HPr with a cysteine point mutation (E5C, E25C or E66C) was mixed with maleimide-EDTA (Toronto Research Chemicals, Cat\# P996250) pre-chelated with $\mathrm{Mn}^{2+}$ for $4 \mathrm{hr}$. The molar ratios of HPr, maleimide-EDTA, and $\mathrm{Mn}^{2+}$ were 1:4:8. The paramagnetically conjugated $\mathrm{HPr}$ was further purified on a Source-Q anion exchange column, and successful ligation was confirmed by electrospray mass spectrometry. 
A TROSY-based pulse scheme was used to measure ${ }^{1} \mathrm{H}_{\mathrm{N}}$ transverse relaxation rates of EIN (300 $\mu \mathrm{M},\left[{ }^{2} \mathrm{H},{ }^{15} \mathrm{~N}\right]$-labeled) mixed with equimolar unlabeled wild-type HPr (diamagnetic) or paramagnetically labeled HPr, to obtain the paramagnetic relaxation enhancement (PRE; $\left.\Gamma_{2}\right)$, as previously described. ${ }^{2}$ The relaxation delay times were set at 8,20 , and $32 \mathrm{~ms}$. The PRE measurements were performed in the buffer or in the presence of the macromolecular crowders. The PREs were calculated based on the structure of the EIN-HPr native complex (PDB entry 3EZA) as described previously, ${ }^{2,3}$ with the $\mathrm{Mn}^{2+}$ electronic relaxation time at $10 \mathrm{~ns}$ and the rotational correlation time of the protein complex at $14 \mathrm{~ns}$.

\section{Measurement of off-resonance $R_{1 \rho}$ rates}

The sample contained $300 \mu \mathrm{M}\left[{ }^{2} \mathrm{H},{ }^{15} \mathrm{~N}\right]$-labeled HPr and $300 \mu \mathrm{M}$ unlabeled EIN. The offresonance $R_{1 \rho}$ rate constants for ${ }^{1} \mathrm{H}_{\mathrm{N}}$ were measured using a pulse sequence described previously. ${ }^{4}$ The spectra were recorded on both $600 \mathrm{MHz}$ and $950 \mathrm{MHz}$ spectrometers. A recycling delay of $2 \mathrm{~s}$ was used between scans, and 8 transients were recorded per $t_{1}$ increment. The carrier of the off-resonance spin-lock field was adjusted relatively to the proton center $(8.2$ ppm) according to $\Delta= \pm \sqrt{ } 2 \omega_{1}$ (the strength of the rf field), while the strength of the spin-lock field $\left(\omega_{1}^{\text {eff }} / 2 \pi\right)$ was varied from 3.3 to $17.7 \mathrm{kHz}(3.3,4.5,6.2,9.1,12.6,17.7 \mathrm{kHz})$. For each spin-lock field, the peak intensities at time delays of $0.005,0.02,0.04,0.06$, and $0.08 \mathrm{~s}$ were fit to a single-exponential decay; the errors for $R_{1 \rho}$ were the uncertainties of the fits. The $R_{1 \rho}$ data collected at the two magnetic fields were globally fit to the following equation for a twostate fast-exchange model: ${ }^{5}$

$$
R_{1 \rho} / \sin ^{2} \theta=\bar{R}_{2}+\bar{R}_{1} / \tan ^{2} \theta+p_{\mathrm{f}} \cdot p_{\mathrm{b}} \cdot\left(\omega_{\mathrm{f}}-\omega_{\mathrm{b}}\right)^{2} \cdot \frac{k_{\mathrm{ex}}}{\left(k_{\mathrm{ex}}\right)^{2}+\left(\omega_{1}^{\mathrm{eff}}\right)^{2}}
$$

Here $\bar{R}_{2}$ and $\bar{R}_{1}$ are the relaxation rates in the rotating frame, with the sum, $\bar{R}_{2}+\bar{R}_{1} / \tan ^{2} \theta$, as a fitting parameter; $\theta$ is the tilt angle of the rotating frame $\left(\sim 35^{\circ}\right) ; p_{\mathrm{f}}$ and $p_{\mathrm{b}}$ stand for the fractions of free and bound HPr, respectively (calculated from $K_{\mathrm{D}}$ determined by CSPs as in 
Figure 1); and $\left(\omega_{\mathrm{f}}-\omega_{\mathrm{b}}\right)$ corresponds to the proton chemical shift perturbation upon complex formation (with its value at $950 \mathrm{MHz}$ set to be $950 / 600$, or 1.58 -fold of that at $600 \mathrm{MHz}$ ). The errors for $k_{\text {ex }}$ were the uncertainties of the fits (reported in Table 1), whereas the errors of $k_{\mathrm{a}}$ and $k_{\mathrm{d}}$ were propagated from the measured $k_{\mathrm{ex}}$ and $K_{\mathrm{D}}$ values.

\section{Calculation of EIN-HPr association rate constant in dilute condition}

The structure of the native complex found in PDB entry $3 \mathrm{EZA}^{6}$ has the side chain of F48 deeply inserted into a crevice on the EIN surface. This F48 conformer led to serious clashes with EIN when we initially applied the TransComp method to determine the transient complex. Because F48 adopts its native conformer only after the formation of the transient complex, we rebuilt the F48 side chain in UCSF Chimera, ${ }^{7}$ by picking a $\operatorname{rotamer}^{8}$ that has the side chain retracted from and also avoids clashes with EIN.

The modified PDB file of the complex was uploaded to the pdb portal of the TRANSCOMP web server (https://pipe.rcc.fsu.edu/transcomp/frompdb.html). ${ }^{9}$ The server converted the pdb file to pqr format using pdb2pqr, ${ }^{10}$ with atomic partial charges assigned for $\mathrm{pH}=7.4$ and atoms assigned the Bondi radii. The server then sent the data to the computer clusters at Florida State University's Research Computing Center to do the calculations. The calculations involved three steps: determination of the transient complex by mapping the interaction energy surface via sampling the 6-dimensional space of relative translation and relative rotation; finding the basal rate constant by Brownian dynamics simulations; and computing the electrostatic interaction energy in the transient complex by the APBS method. ${ }^{11}$ Sample results at the ionic strength of $0.17 \mathrm{M}$ and temperature of $298 \mathrm{~K}$ are available at https://pipe.rcc.fsu.edu/transcomp/out/32a396b47d2832507b57017643025bd2/4689/. The final results were corrected to the experimental temperature of $313 \mathrm{~K}$. The error of the basal rate constant $\left(k_{\mathrm{a} 0}\right)$ was estimated by assuming a binomial distribution for the fates (whether surviving the binding reaction) of 4000 Brownian trajectories. The error of the association rate 
constant was estimated by computing the error of the basal rate constant and the error of the electrostatic interaction energy in the transient complex; the latter was taken as the standard error of the mean, calculated from the results in 100 transient-complex configurations.

\section{Calculation of the crowder-induced change in EIN-HPr interaction energy}

Our FMAP method (FFT-based Modeling of $\underline{\text { Atomistic }}$ Protein-crowder interactions) was developed to calculate the transfer free energy of a protein from a dilute solution to a crowded solution. ${ }^{12}$ In this method, protein-crowder interactions (including steric, nonpolar, and electrostatic terms) are expressed as correlation functions, which are a direct product of two functions in Fourier space. The weighting factor for the nonpolar term was 0.16; the weighting factor for the electrostatic term was 1.6 ; the cutoff distance was $12 \AA$. The ion strength was $0.17 \mathrm{M}$, and the temperature was set to $313 \mathrm{~K}$.

To prepare the crowded solution, BSA was built by homology modeling using a human serum albumin structure (PDB entry 1AO6) and eight copies were placed in a cubic box with a side length of $199.423 \AA$ (concentration at $111 \mathrm{~g} / \mathrm{L}$ ) and simulated in explicit solvent for 70 ns. ${ }^{12}$ The eight copies of BSA in the final snapshot was saved as representing the crowded solution. For each test protein (e.g., HPr), the Boltzmann factor of the protein-crowder interaction energy was averaged over $334 \times 334 \times 334$ grid points and over 4392 orientations of the protein, to yield the transfer free energy, $\Delta \mu$, from a dilute solution to the crowded solution. For each EIN-HPr complex, this calculation was performed three times, once for the complex ("C") and once for each of the two partner proteins ("A" and "B"). The difference, $\Delta \mu_{\mathrm{C}}-\left(\Delta \mu_{\mathrm{A}}+\Delta \mu_{\mathrm{B}}\right)$, is finally the crowder-induced change in EIN-HPr interaction energy.

This calculation was done for the native complex to yield $\Delta \Delta G_{\mathrm{c}}^{\mathrm{b}}$, the crowding effect of BSA on the binding free energy, and also when the two partner proteins were separated to a given distance, to yield $\Delta \Delta G_{\mathrm{c}}$ as a function of the inter-protein separation. In the latter case, the 
two partner proteins were separated along the normal direction of the least-squares plane of the interfacial atoms. ${ }^{9}$ If the resulting configuration had clashes between the partner proteins (which could happen when the separation was $<\sim 4 \AA$ ), we replaced it with the closest one sampled by TransComp. The selection for the closest configuration was based on root-meansquare-deviation.

\section{Residue-level decomposition of BSA-EIN and BSA-HPr interaction energies}

Energy decomposition was carried out by considering the interaction of a test protein, e.g., HPr, with a single BSA molecule (taken as copy \#1 in the crowder box), using our FMAPB23 method. ${ }^{13}$ We selected the 1000 HPr-BSA pair configurations with the lowest interaction energies according to FMAPB23, and then recalculated their interaction energies by the atombased method (which finds the interaction energy by enumerating all pairs of atoms between the protein-crowder pair). For each HPr-BSA pair configuration, the interactions between all the atom pairs connecting one residue of the protein with one residue of the crowder were added up, and this partial sum was divided by two, with one half assigned to the protein residue and one half assigned to the crowder atom. The total contribution of each protein residue was accumulated over all the crowder residues as interaction partners, and a final average over the 1000 protein-crowder configurations was taken. This calculation was done both on the EINHPr native complex, yielding the residue-level decomposition in the bound state, and on the two partner proteins, yielding the decomposition in the free state. 


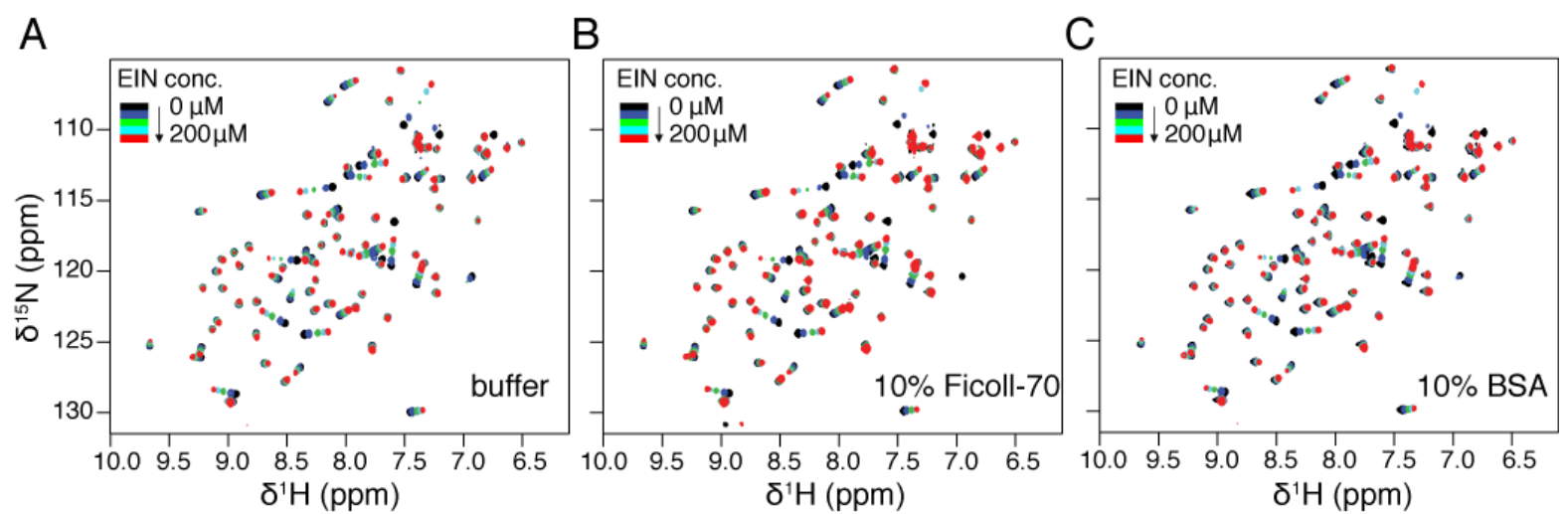

Figure S1. Overlay of the HSQC spectra of $100 \mu \mathrm{M}{ }^{15} \mathrm{~N}$-labeled HPr at increasing concentrations of unlabeled EIN. The HSQC spectra were collected in (A) the buffer $(20 \mathrm{mM}$ Tris $\bullet \mathrm{HCl}$ at $\mathrm{pH} 7.4$ with $150 \mathrm{mM} \mathrm{NaCl}$ ), (B) $10 \%$ w/v Ficoll-70, or (C) $10 \%$ w/v BSA. The titration of EIN into HPr caused CSPs in the same set of residues along the same directions, regardless of the solution conditions. 


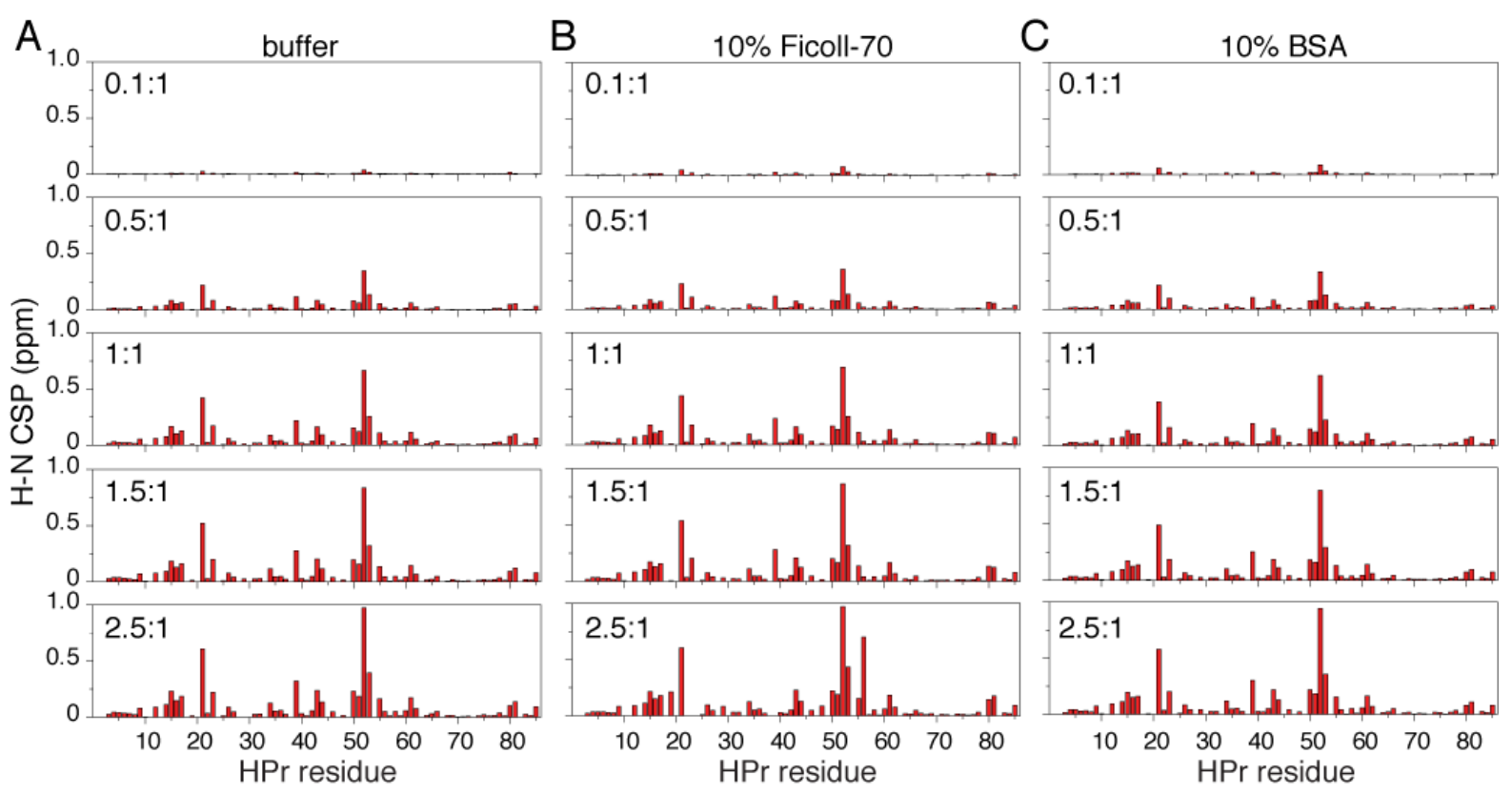

Figure S2. CSP profiles for $100 \mu \mathrm{M}{ }^{15} \mathrm{~N}$-labeled HPr upon the addition of unlabeled EIN. The CSPs were measured in (A) the buffer, (B) 10\% w/v Ficoll-70, or (C) 10\% w/v BSA. 

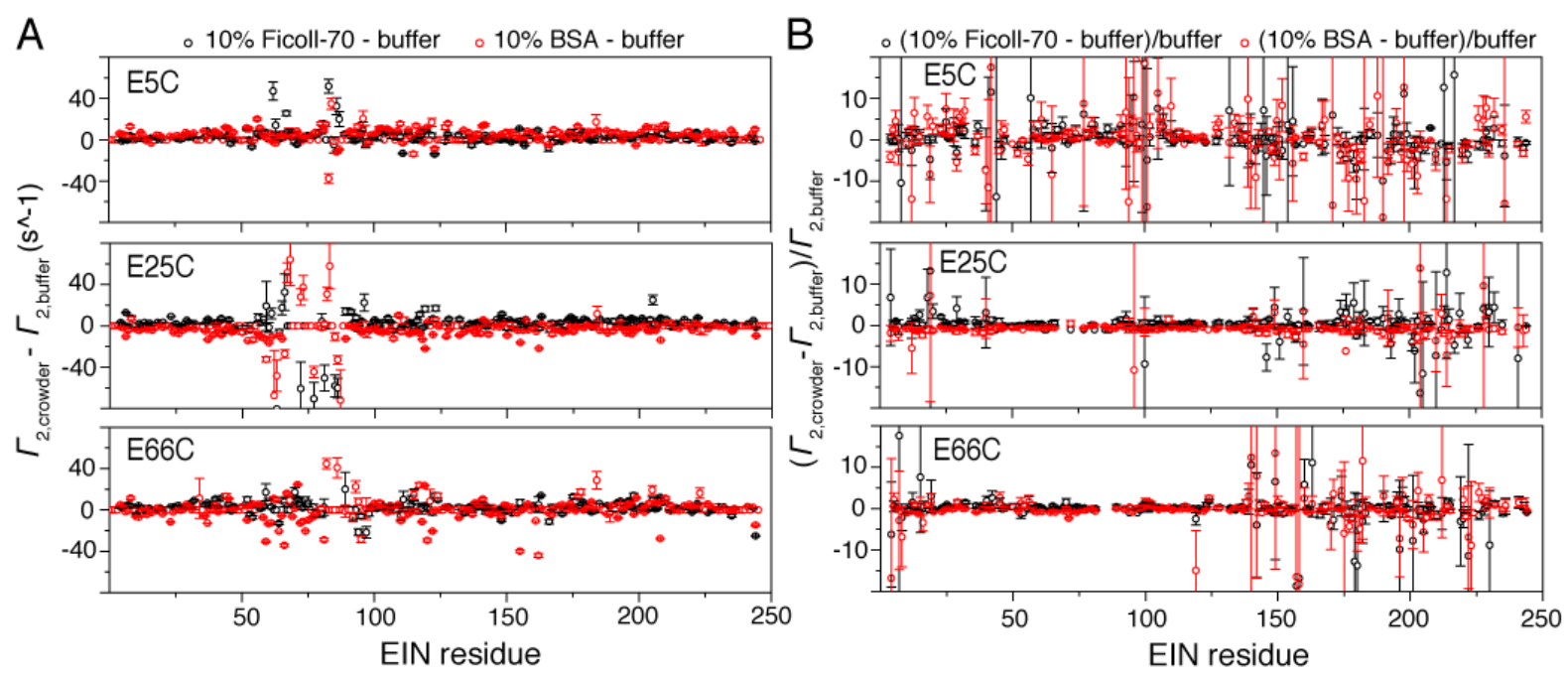

Figure S3. The difference in the intermolecular PRE $\Gamma_{2}$ values for backbone ${ }^{1} \mathrm{H}_{\mathrm{N}}$ collected in the diluted buffer or in the presence of macromolecular crowders. (A) The absolute differences in the intermolecular PRE $\Gamma_{2}$ values. The PRE values in the crowded conditions are multiplied by a factor $(0.9$ for Ficoll-70 and 1.14 for BSA) before subtraction, to account for the difference in $K_{\mathrm{D}}$ and bound fraction. (B) The relative PRE differences. For each residue, the absolute PRE difference in (A) is further divided by the PRE value observed in the diluted condition. 

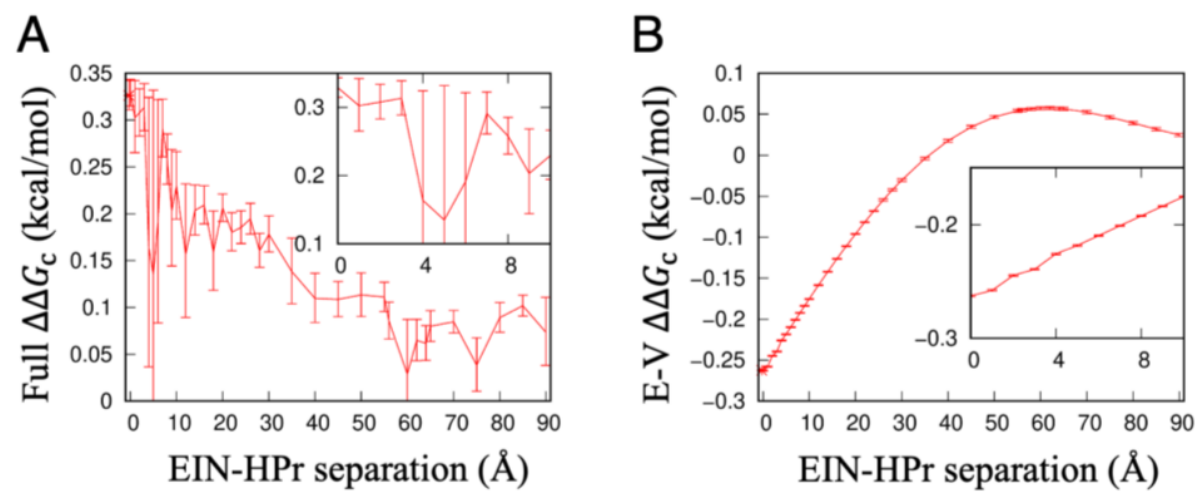

Figure S4. The change, $\Delta \Delta G_{\mathrm{c}}$, in the interaction energy between EIN and HPr by the presence of $10 \%$ w/v BSA. $\Delta \Delta G_{\mathrm{c}}$ is calculated in the same way as $\Delta \Delta G_{\mathrm{c}}^{\mathrm{b}}$ illustrated in Figure 5A, except that EIN and HPr are placed at various separations. (A) $\Delta \Delta G_{\mathrm{c}}$ results when both volume exclusion by and soft interactions with BSA are fully accounted for. (B) $\Delta \Delta G_{\mathrm{c}}$ when only the excluded-volume ("E-V") effect of BSA is considered. Error bars represent variations among FMAP results obtained from 4392 orientations of the protein complex. 


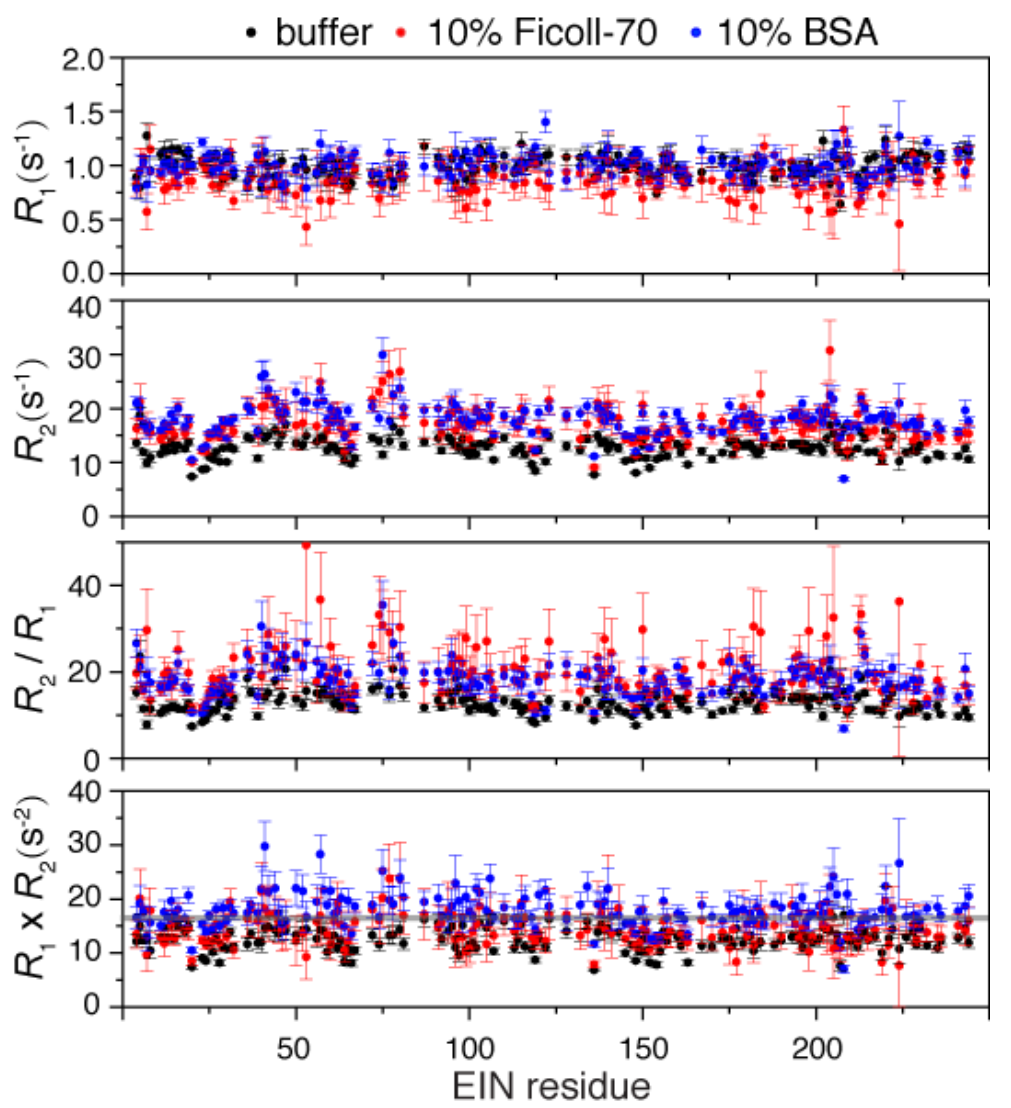

Figure S5. NMR probe of the soft interactions between the protein crowder BSA and free EIN. Longitudinal relaxation rates $R_{1}\left(1^{\text {st }}\right.$ row $)$, transverse relaxation rates $R_{2}$ ( $2^{\text {nd }}$ row), the ratio between $R_{2}$ and $R_{1}$ ( $3^{\text {rd }}$ row), and the product of $R_{1}$ and $R_{2}$ rates ( $4^{\text {th }}$ row), measured for the amide nitrogens of $\left[{ }^{2} \mathrm{H},{ }^{15} \mathrm{~N}\right]$-labeled free EIN in three solutions conditions. The rigid limit of $R_{1} \times R_{2}$, with $\varpi_{N} \times \tau_{c} \gg 1$ and $S^{2}=0.86$, is shown as a gray line. Error bars represent the standard errors of the fits of the peak intensities at different delay times ( $1^{\text {st }}$ and $2^{\text {nd }}$ rows) and propagated errors ( $3^{\text {rd }}$ and $4^{\text {th }}$ rows). 


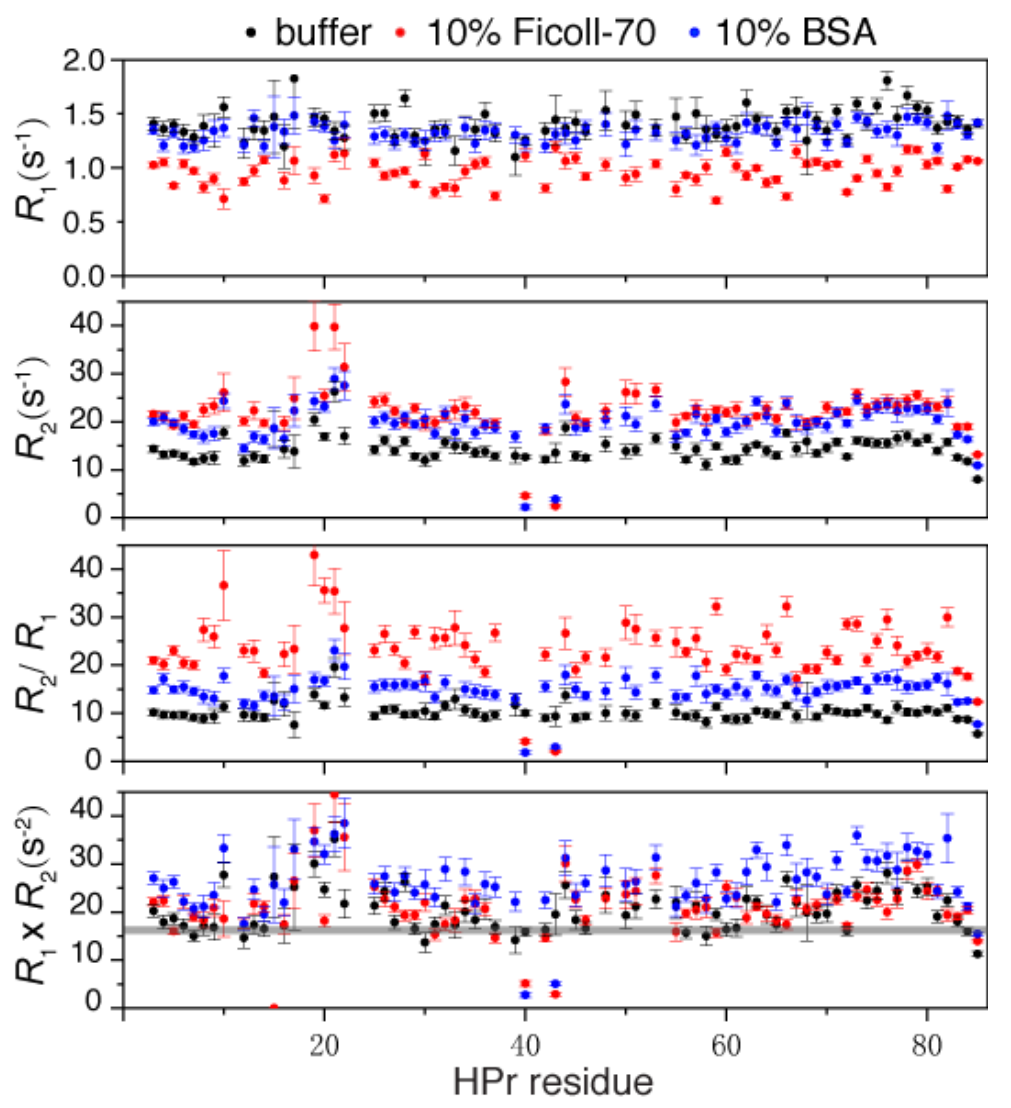

Figure S6. NMR probe of the soft interactions between BSA and HPr in complex with EIN. Longitudinal relaxation rates $R_{1}$ ( $1^{\text {st }}$ row), transverse relaxation rates $R_{2}$ ( $2^{\text {nd }}$ row), the ratio between $R_{2}$ and $R_{1}$ ( $3^{\text {rd }}$ row), and the product of $R_{1}$ and $R_{2}$ rates ( $4^{\text {th }}$ row), measured for the amide nitrogens of $\left[{ }^{2} \mathrm{H},{ }^{15} \mathrm{~N}\right]$-labeled $\mathrm{HPr}$ in complex with equimolar unlabeled EIN at $300 \mu \mathrm{M}$ in three solutions conditions. The rigid limit of $R_{1} \times R_{2}$, with $\varpi_{N} \times \tau_{c} \gg 1$ and $S^{2}=0.86$, is shown as a gray line. Note that $\mathrm{HPr}$ residues interfacing with EIN show elevated $R_{1} \times R_{2}$ values above the rigid-limit line. Errors bar represent the standard errors of the fits of the peak intensities at different delay times ( $1^{\text {st }}$ and $2^{\text {nd }}$ rows $)$ and propagated errors ( $3^{\text {rd }}$ and $4^{\text {th }}$ rows). 


\section{REFERENCES}

1. Delaglio, F.; Grzesiek, S.; Vuister, G. W.; Zhu, G.; Pfeifer, J.; Bax, A. NMRPipe: a multidimensional spectral processing system based on UNIX pipes. J. Biomol. NMR 1995, 6 (3), 277-293, DOI: 10.1007/BF00197809.

2. Iwahara, J.; Tang, C.; Clore, G. M. Practical aspects of ${ }^{1} \mathrm{H}$ transverse paramagnetic relaxation enhancement measurements on macromolecules. J. Magn. Reson. 2007, 184 (2), 185-195, DOI: 10.1016/j.jmr.2006.10.003.

3. Tang, C.; Iwahara, J.; Clore, G. M. Visualization of transient encounter complexes in protein-protein association. Nature 2006, 444 (7117), 383-386, DOI: 10.1038/nature05201.

4. Eichmuller, C.; Skrynnikov, N. R. A new amide proton R1rho experiment permits accurate characterization of microsecond time-scale conformational exchange. J. Biomol. NMR 2005, 32 (4), 281-293, DOI: 10.1007/s10858-005-0658-y.

5. Palmer, A. G.; Massi, F. Characterization of the Dynamics of Biomacromolecules Using RotatingFrame Spin Relaxation NMR Spectroscopy. Chem. Rev. 2006, 106 (5), 1700-1719, DOI: $10.1021 / \mathrm{cr} 0404287$.

6. Garrett, D. S.; Seok, Y. J.; Peterkofsky, A.; Gronenborn, A. M.; Clore, G. M. Solution structure of the 40,000 Mr phosphoryl transfer complex between the N-terminal domain of enzyme I and HPr. Nat. Struct. Biol. 1999, 6 (2), 166-173, DOI: 10.1038/5854.

7. Pettersen, E. F.; Goddard, T. D.; Huang, C. C.; Couch, G. S.; Greenblatt, D. M.; Meng, E. C.; Ferrin, T. E. UCSF Chimera--a visualization system for exploratory research and analysis. J. Comput. Chem. 2004, 25 (13), 1605-1612, DOI: 10.1002/jcc.20084.

8. Lovell, S. C.; Word, J. M.; Richardson, J. S.; Richardson, D. C. The penultimate rotamer library. Proteins 2000, 40 (3), 389-408, DOI: https://doi.org/10.1002/1097-0134.

9. Qin, S.; Pang, X.; Zhou, H. X. Automated prediction of protein association rate constants. Structure 2011, 19 (12), 1744-1751, DOI: 10.1016/j.str.2011.10.015.

10. Dolinsky, T. J.; Nielsen, J. E.; McCammon, J. A.; Baker, N. A. PDB2PQR: an automated pipeline for the setup of Poisson-Boltzmann electrostatics calculations. Nucleic Acids Res. 2004, 32 (Web Server issue), W665-667, DOI: 10.1093/nar/gkh381.

11. Baker, N. A.; Sept, D.; Joseph, S.; Holst, M. J.; McCammon, J. A. Electrostatics of nanosystems: application to microtubules and the ribosome. Proc. Natl. Acad. Sci. U.S.A. 2001, 98 (18), 10037-10041, DOI: $10.1073 /$ pnas.181342398.

12. Qin, S.; Zhou, H. X. Further Development of the FFT-based Method for Atomistic Modeling of Protein Folding and Binding under Crowding: Optimization of Accuracy and Speed. J. Chem. Theory Comput. 2014, 10 (7), 2824-2835, DOI: $10.1021 / \mathrm{ct} 5001878$.

13. Ahn, S. H.; Qin, S.; Zhang, J. Z.; McCammon, J. A.; Zhang, J.; Zhou, H. X. Characterizing protein kinase A (PKA) subunits as macromolecular regulators of PKA RIalpha liquid-liquid phase separation. J. Chem. Phys. 2021, 154 (22), 221101, DOI: 10.1063/5.0049810. 